\title{
Comparing the Importance of Hemodynamic Obstruction with Diastolic Dysfunction in Patients with Hypertrophic Obstructive Cardiomyopathy
}

\author{
David A. D'Alessandro ${ }^{1}$, Edvard Skripochnik ${ }^{1}$, Robert E Michler ${ }^{1}$, Viktoria Hentschel ${ }^{2}$ and \\ Siyamek Neragi-Miandoab ${ }^{*}, 1$
}

\author{
${ }^{I}$ Department of Cardiovascular and Thoracic Surgery, Montefiore Medical Center, Albert Einstein College of Medicine, NY, USA \\ ${ }^{2}$ University of Bonn, Bonn, Germany
}

\begin{abstract}
Objective: Myomectomy is the cornerstone of therapy for hypertrophic obstructive cardiomyopathy (HOCM) in the presence of a high gradient. The importance of mechanical gradient across the left ventricle outflow track (LVOT) $v s$ left ventricular diastolic dysfunction (LVDD) is debatable.

Methods: We retrospectively analyzed data on 14 patients with HOCM who underwent myomectomy from 2007 to 2011 at our institution. All patients in this study were symptomatic. The purpose of this study was to assess the significance of immediate reduction of the gradient across the LVOT as well as improved LVDD and its correlation with hemodynamics.

Results: A total of 14 patients with a mean age of $52.5 \pm 19.0$ years (male-female ratio of 5/8) were evaluated. The preoperative LVOT peak gradient was $76.9 \pm 63.4 \mathrm{mmHg}$, the left atrial (LA) diameter was $41.9 \pm 6.1 \mathrm{~mm}$, and the septal thickness was $15.4 \pm 3.2 \mathrm{~mm}$. The relevant preoperative risk factors included DM $(23.0 \% ; n=3)$, angina pectoris $(15.4 \%$; $n=2)$, cerebrovascular disease (CVD) $(30.8 \% ; n=4)$, stroke $(15.4 \% ; n=2)$, arrhythmias $(30.8 \% ; n=4)$, and COPD $(15.4 \% ; n=2)$. The concurrent procedures included mitral valve repair/ replacement (MVR) $(30.8 \% ; n=4)$, aortic valve replacement (AVR) $(23.0 \% ; n=3)$, coronary artery bypass grafting (CABG) $(15.4 \% ; n=2)$, and modified MAZE procedure/ablation $(15.4 \% ; n=2)$. The perioperative mortality was $7.7 \%(n=1)$, and the long-term survival was $85.6 \%$ at a median follow up of 30 months. The postoperative LVOT gradient improved to $32.3 \pm 24.4 \mathrm{mmHg}$ and the septal thickness to $12.5 \pm 3.8 \mathrm{~mm}$. These differences were not statistically significant, likely due to small sample size. The postoperative complications included iatrogenic small VSD in one patient (who had myomectomy for a third time), atrial fibrillation $(n=4)$, cardiac arrest $(7.7 \% ; n=1)$, neurologic adverse event $(7.7 \% ; n=1)$, and new onset renal failure $(7.7 \%$; $n=1)$. We did not observe any new onset AV block. The length of stay (LOS) in the surgical critical care unit was $95.5 \pm$ 112.7 hours. The overall hospital LOS was $15 \pm 11.5$ days.

Conclusion: The septal myomectomy results showed an immediate reduction of the LVOT gradient, which translates into clinical and echocardiographic improvement.
\end{abstract}

Keywords: HOCM, LVOT, myomectomy, left ventricle diastolic dysfunction, diastolic dysfunction.

\section{INTRODUCTION}

Hypertrophic cardiomyopathy is a complex genetic disease [1] with a prevalence of 1 in 500 in the general population [2]. Although HOCM is the most frequent cause of sudden death in young people, the majority of affected individuals remain undiagnosed for a long time [3, 4]. Some patients with symptomatic hypertrophic cardiomyopathy have minimal LVOT gradients, and there is uncertainty as to whether their symptoms are due to LVDD or a high gradient across the LVOT. The purpose of this study was to characterize the immediate response to myomectomy, which may indicate that LVOT obstruction was paramount to clinical and echocardiographic presentation of HOCM

*Address correspondence to this author at the Department of Cardiovascular and Thoracic Surgery, Montefiore Medical Center, Albert Einstein College of Medicine, 3400 Bainbridge Ave, MAP 5, New York, NY, 10467, USA; Tel: 001646872 6765; Fax: 001718920 8556;

E-mail: sneragi@yahoo.com patients and that LVDD may not be as crucial as has previously been assumed.

\section{METHODS}

We retrospectively analyzed data on 14 patients with HOCM who underwent myomectomy between 2007 and 2011 at our institution. All patients in this study were symptomatic despite medical therapy and had significant gradient across the LVOT at rest or inducible with the valsalva maneuver or medication (isoproterenol). All operations were performed using a standard approach with a median sternotomy, extracorporeal circulation, and transaortic approach. Preoperative transthoracic, intraoperative transesophageal, and postoperative transthoracic echocardiography was performed to assess LVOT diameter and gradients, septal thickness, mitral valve function. 


\section{STATISTICAL ANALYSIS}

Patients' demographics, risk factors, and postoperative outcome data were collected. Hospital mortality was defined as death for any reason occurring within 30 days after the operation or any time during the same hospitalization, regardless of the length. Survival curves were generated using the Kaplan-Meier method (Fig. 1). For continuous variables, correlations were calculated with the Student $t$ test. Data analysis was performed with the Graphpad Prism program.

\section{RESULTS}

A total of 14 patients with a mean age of $52.5 \pm 19$ years (male-female ratio of 5/8) were evaluated. The majority of patients $(n=11)$ were hypertensive; 2 patients had stable angina pectoris, and 4 patients had chronic arrhythmias. Most of the patients had compromised LV function with a mean NYHA class of $3 \pm 0.96$. Table 1 shows the preoperative characteristics of the patients. The preoperative LVOT peak gradient was $76.9 \pm 63.4 \mathrm{mmHg}$, the LA diameter was $41.9 \pm 6.1 \mathrm{~mm}$, and the septal thickness was

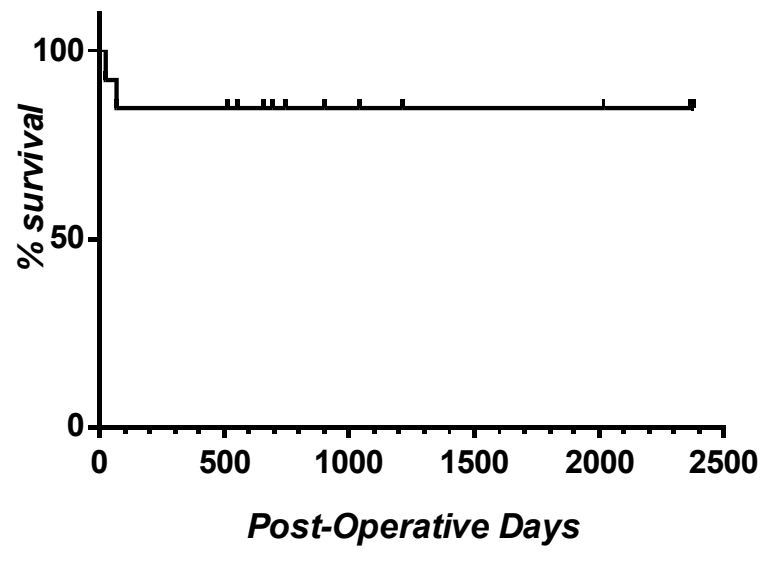

Fig. (1). Kaplan-Meier estimated survival curve.

$15.4 \pm 3.2 \mathrm{~mm}$. Two patients had had previous cardiac surgery (15.4\%); one of them had had previous MVR/AVR, and the other had had a repair of ascending aorta and an AICD implantation. A total of 2 patients $(15.4 \%)$ had preoperative AICD in place. The concomitant procedures included MVR, AVR, CABG, and ablation. Table 2 shows the perioperative characteristics of the patients. One patient

Table 1. Preoperative Characteristics of Patients

\begin{tabular}{|c|c|c|c|c|}
\hline Age $(y)$ & - & - & $52.5 \pm 19.0$ & 58 \\
\hline Sex (male/female) & $5 / 8$ & $38.5 / 61.5$ & - & - \\
\hline Body Surface Area $\left(\mathrm{m}^{2}\right)$ & - & - & $1.89 \pm 0.2$ & 1.92 \\
\hline Hypertension & 11 & 84.6 & - & - \\
\hline Hypercholesterolemia & 3 & 23.0 & - & - \\
\hline Smoking & 3 & 23.0 & - & - \\
\hline Family History (Coronary Artery Disease) & 3 & 23.0 & - & - \\
\hline Cerebrovascular Disease & 4 & 30.8 & - & - \\
\hline Cerebrovascular Accident & 2 & 15.4 & - & - \\
\hline Peripheral Vascular Disease & 0 & 0 & - & - \\
\hline Arrhythmia & 4 & 30.8 & - & - \\
\hline Asthma/ COPD & 2 & 15.4 & - & - \\
\hline Pulmonary Hypertension & 1 & 7.7 & - & - \\
\hline Cardiovascular Failure & - & 38.5 & - & - \\
\hline - NYHA Stage & & & & III \\
\hline
\end{tabular}


Table 2. Perioperative Parameters

\begin{tabular}{|c|c|c|c|c|}
\hline $\begin{array}{l}\text { Characteristics } \\
\text { - Operative Procedure - }\end{array}$ & Number (n) & Percentage (\%) & $\begin{array}{c}\text { Mean } \pm \text { Standard } \\
\text { Deviation }\end{array}$ & Median \\
\hline Mitral Valve Repair/ Replacement & 4 & 30.8 & - & - \\
\hline Aortic Valve Repair/ Replacement & 3 & 23.0 & - & - \\
\hline Coronary Artery Bypass Grafting & 2 & 15.4 & - & - \\
\hline Ablation & 2 & 15.4 & - & - \\
\hline Perfusion Time (min) & - & - & $130.3 \pm 60.3$ & 145 \\
\hline Aortic Cross Clamp Time (min) & - & - & $81.1 \pm 44.3$ & 69 \\
\hline Longest Ischemic Interval (min) & - & - & $28.1 \pm 15.6$ & 23 \\
\hline Total Volume of Cardioplegic Solution $(\mathrm{ml})$ & - & - & $2712 \pm 1631.2$ & 3175 \\
\hline Hematocrit after Cardiopulmonary Bypass & - & - & $25.7 \pm 3.6$ & 26.0 \\
\hline Red Blood Cell Units & 3 & 23.0 & - & - \\
\hline Platelet Units & 2 & 15.4 & - & - \\
\hline Fresh Frozen Plasma Units & 2 & 15.4 & - & - \\
\hline Cryoprecipitate Units & 1 & 7.7 & - & - \\
\hline Highest Lactate $(\mathrm{mmol} / \mathrm{L})$ & - & - & $1.95 \pm 0.8$ & 1.7 \\
\hline Glucose $(m g / d L)$ & & & & \\
\hline
\end{tabular}

died perioperatively (7.7\%); this patient had suffered from preoperative angina and advanced CHF. At a median follow up, another patient had expired from a non-cardiac-related condition. This patient had hepatitis C, DM, CHF, persistent HTN, and prostate cancer. The long-term survival was $85.6 \%$ at a median follow up of 30 months.

Echocardiographic evaluation in the preoperative period and postoperative prior to discharge from the hospital demonstrated the following: (a) the mean LVOT peak gradient $(\mathrm{mmHg})$ improved from $76.9 \pm 63.4$ to $32.3 \pm 24.4$ postoperatively $(p=.1612)$; (b) the septal thickness decreased from $15.4 \pm 3.2 \mathrm{~mm}$ to $12.5 \pm 3.8 \mathrm{~mm}$ postoperatively ( $p=.0984)$; and (c) the LA diameter $(41.9 \pm$ $6.1 \mathrm{~mm}$ ) remained relatively constant postoperatively $(40.5 \pm$ $11.1 ; p=.17$ ) (not statistically significant). The mitral valve demonstrated only mild regurgitation in 5 patients (38.5\%) postoperatively. Table 3 demonstrates the echocardiographic data preoperative and postoperatively. The postoperative complications included atrial fibrillation $(30.8 \% ; n=4)$, need for reoperation $(15.4 \% ; n=2)$, neurologic adverse event $(7.7 \% ; n=1)$, and new onset renal failure $(7.7 \%$; $n=1$ ). One patient developed a small VSD postoperatively, which was hemodynamically not significant. This patient had undergone two previous myomectomies yet had persistently elevated LVOT gradient. In the perioperative period, one patient developed new onset renal failure; this patient had compromised kidney function preoperatively. We did not observe any new onset AV block. The mean postoperative ventilation time was $48.3 \pm 94.1$ hours, and
LOS in the critical care unit was $95.5 \pm 112.7$ hrs. Table 4 demonstrates the postoperative outcomes. The hospital LOS was $15.0 \pm 11.5$ days. Of the 13 surviving patients, 8 patients were discharged home and 3 patients were discharged to skilled nursing and rehabilitation facilities.

\section{DISCUSSION}

An elevated gradient across the LVOT is the major culprit in symptomatic HOCM patients [5], mandating a surgical myomectomy in an attempt to lower the gradient to non-obstructive levels and here $[1,4-6]$ and to reduce the LV mass index [6]. Consequently, the relief of LVOT obstruction causes immediate improvement in symptoms following myomectomy [7]. As expected, we observed this direct improvement of LOVT gradient in our series, which was demonstrated by perioperative echocardiograms.

LVDD also contributes to patients' symptoms and LV compromise but may require a longer recovery period after the LVOT obstruction has been released. It has been reported [8] that preoperative and postoperative wall thickness as well as LV hypertrophy (which is an indication of LVDD) are not associated with increased risk of postoperative mortality. As such, we believe that symptomatic relief following myomectomy is a result of reduced gradient across LVOT and less likely due to improved LVDD. This may indicate that current medical management of HOCM is not adequately targeting the main issue. Medical management focuses on improving the LVDD, which may not affect the 
Table 3. Per- and Postoperative Echocardiographic Findings

\begin{tabular}{|c|c|c|c|c|c|c|c|}
\hline Echocardiographical Characteristics & $\begin{array}{c}\text { Number } \\
\text { (n) }\end{array}$ & $\begin{array}{l}\text { Percentage } \\
(\%)\end{array}$ & \multicolumn{2}{|c|}{ Pre-Operative } & \multicolumn{2}{|c|}{ Post-Operative } & $\mathbf{p}$ \\
\hline Left Ventricular Outflow Tract Peak Gradient $(\mathrm{mmHg})$ & - & - & $76.9 \pm 63.4$ & 80.4 & $32.3 \pm 24.4$ & 22.5 & 0.16 \\
\hline Left Atrium Ø $(\mathrm{mm})$ & - & - & $41.9 \pm 6.1$ & 42 & $40.5 \pm 11.1$ & 41.5 & 0.17 \\
\hline Septal Thickness $(\mathrm{mm})$ & - & - & $15.4 \pm 3.2$ & 14.5 & $12.5 \pm 3.8$ & 11 & 0.09 \\
\hline Post-Operative Mitral Regurgitation, mild & 5 & 38.5 & \multicolumn{2}{|l|}{-} & \multicolumn{2}{|l|}{-} & - \\
\hline Post-Operative Ventricular Septum Defect & 1 & 7.7 & \multicolumn{2}{|l|}{-} & \multicolumn{2}{|l|}{-} & - \\
\hline
\end{tabular}

Table 4. Postoperative Complications

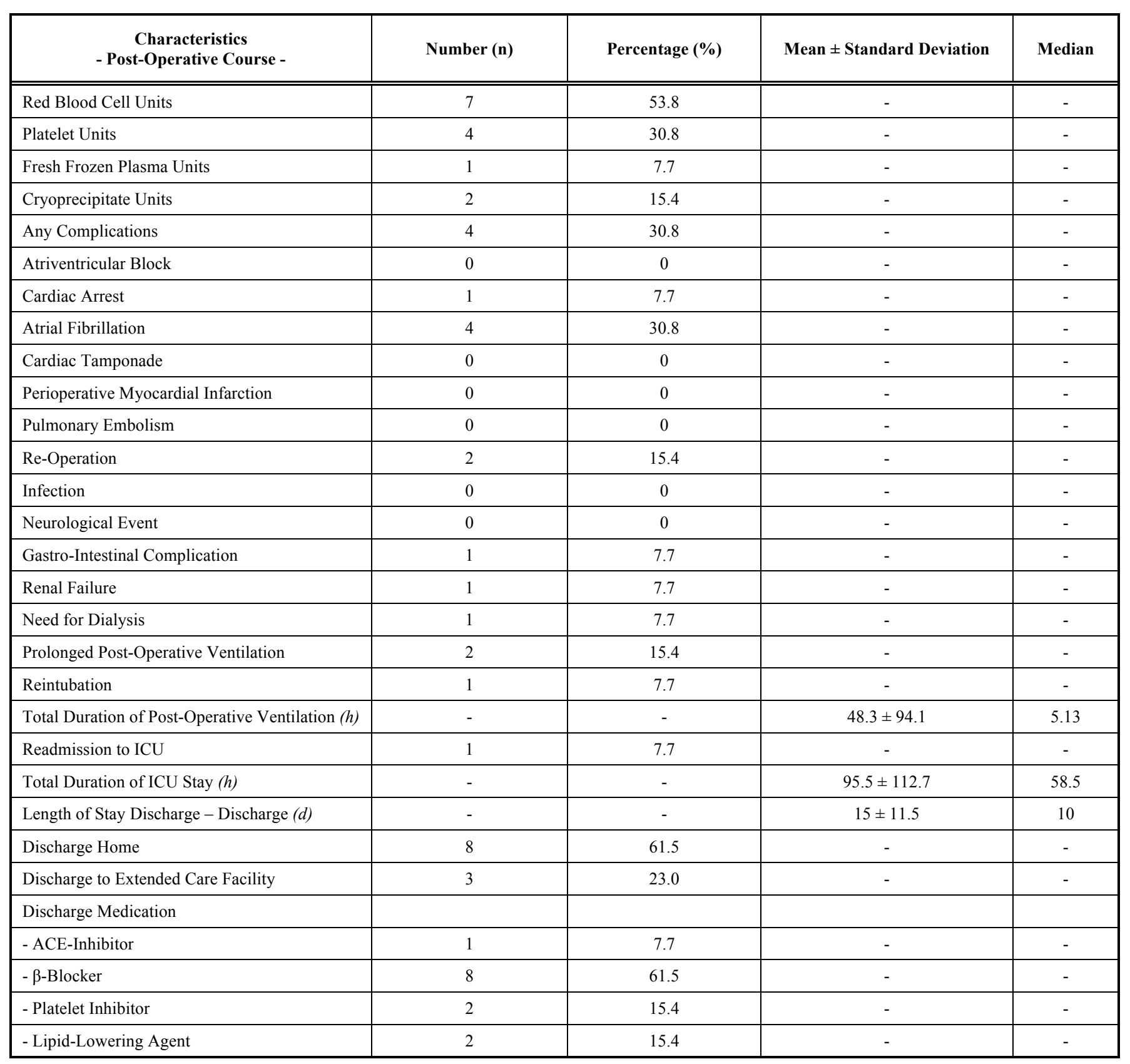


LVOT gradient and hence would not improve symptoms or survival rate. A surgical approach remains the gold standard $[1,4,6]$ particularly in younger patients, while alcohol septal ablation (ASA) is recommended for older patients and those with serious comorbidities $[2,5]$ or mid-ventricular obstruction [1]. Unlike surgical myomectomy, ASA creates an intra-myocardial scar that may potentiate the risk of ventricular arrhythmias and sudden cardiac death $[9,10]$.

Our series showed several new findings in the postoperative setting involving iatrogenic malformations and improvements. A patient of ours required repeated septal myomectomy due to recurrent elevated LVOT gradient and developed a VSD after the third procedure. Although iatrogenic VSD is very rare [6], repeated myomectomy may increase the risk. Our finding may contradict prior case scenarios in the literature, in which recurrent elevated peak gradients and septal hypertrophy was not shown to develop progressive disease [5]. Postoperative conduction system abnormalities are one of the most frequent complications following myomectomy [6-8]. Brown et al. [8] reported a $17 \%$ rate of preoperative automated implantable cardioverter defribillator (AICD). In our series, 1 patient had a previous AICD implantation and none of our patients required new postoperative AICD or PPM implantation. Wang et al. [7] reported a significant improvement of mitral valve regurgitation (MR) postoperatively. MR improved following myomectomy in our series as well but was not statistically significant.

The survival rate of patients following surgical myomectomy is comparable to that of an age- and gendermatched population. In a large series of 416 patients with HOCM who underwent myomectomy reported a favorable survival of $99 \%, 97 \%$, and $85 \%$ at 1,5 , and 10 years, respectively. Our population and long-term survival were similar these previous findings $[8,11]$. The main goal of our study was to compare the significance of LVOT gradient and LVDD reduction. In our limited patient-set we have highlighted a focus for a larger series, which may validate the significance of our observation.

\section{SUMMARY}

Septal myomectomy can be performed with acceptable perioperative mortality and postoperative complications. An immediate reduction of the LVOT gradient can be achieved. These rapid clinical and radiographic improvements suggest that a dynamic obstruction of the LVOT is the major hemodynamic problem as opposed to LVDD.

\section{CONFLICT OF INTEREST}

The authors confirm that this article content has no conflict of interest.

\section{ACKNOWLEDGEMENTS}

Declared none.

\section{REFERENCES}

[1] Knyshov G, Lazoryshynets V, Rudenko K, et al. Is surgery the gold standard in the treatment of obstructive hypertrophic cardiomyopathy? Interact Cardiovasc Thorac Surg 2013; 16(1): 59.

[2] Elmariah S, Fifer MA. Medical, surgical and interventional management of hypertrophic cardiomyopathy with obstruction. Curr Treat Opt Cardiovasc Med 2012; 14(6): 665-78.

[3] Maron BJ, Maron MS. Hypertrophic cardiomyopathy. Lancet 2013; 381(9862): 242-55.

[4] Di Tommaso L, Stassano P, Mannacio V, et al. Asymmetric septal hypertrophy in patients with severe aortic stenosis: The usefulness of associated septal myomectomy. J Thorac Cardiovasc Surg 2013;145(1): 171-5.

[5] Hickey EJ, McCrindle BW, Larsen SH, et al. Hypertrophic cardiomyopathy in childhood: disease natural history, impact of obstruction, and its influence on survival. Ann Thorac Surg 2012; 93: 840-8.

[6] Kayalar N, Schaff HV, Daly RC, Dearani JA, Park SJ. Concomitant septal myomectomy at the time of aortic valve replacement for severe aortic stenosis. Ann Thorac Surg 2010; 89: 459-64.

[7] Wang S, Luo M, Sun $\mathrm{H}$, et al. A retrospective clinical study of transaortic extended septal myomectomy for obstructive hypertrophic cardiomyopathy in China. Eur J Cardiothorac Surg 2013; 43(3): 534-40.

[8] Brown ML, Schaff HV, Dearani JA, Li Z, Nishimura RA, Ommen SR. Relationship between left ventricular mass, wall thickness, and survival after subaortic septal myomectomy for hypertrophic obstructive cardiomyopathy. J Thorac Cardiovasc Surg 2011; 141: 439-43.

[9] Leonardi RA, Kransdorf EP, Simel DL, Wang A. Meta-analyses of septal reduction therapies for obstructive hypertrophic cardiomyopathy: comparative rates of overall mortality and sudden cardiac death after treatment. Circ Cardiovasc Interv 2010; 3: 97104.

[10] Fifer MA, Sigwart U. Controversies in cardiovascular medicine. Hypertrophic obstructive cardiomyopathy: alcohol septal ablation. Eur Heart J 2011; 32: 1059-64.

[11] Schaff HV, Dearani JA, Ommen SR, Sorajja P, Nishimura RA Expanding the indications for septal myomectomy in patients with hypertrophic cardiomyopathy: results of operation in patients with latent obstruction. J Thorac Cardiovasc Surg 2012; 143: 303-9. 